\title{
Inter-Active Site Communication Mediated by the Dimer Interface Beta-Sheet in the Half-the-Sites Enzyme, Thymidylate Synthase.
}

\author{
Paul J. Sapienza ${ }^{1,{ }^{*}}$, Konstantin I. Popov ${ }^{2}$, David D. Mowrey², Bradley T. Falk ${ }^{2}$, Nikolay V. \\ Dokholyan $^{3,4}$, Andrew L. Lee ${ }^{1,2}$ \\ ${ }^{1}$ Division of Chemical Biology and Medicinal Chemistry, Eshelman School of Pharmacy, \\ University of North Carolina at Chapel Hill \\ ${ }^{2}$ Department of Biochemistry and Biophysics, School of Medicine, University of North Carolina at \\ Chapel Hill \\ ${ }^{3}$ Department of Pharmacology, Department of Biochemistry \& Molecular Biology, Penn State \\ College of Medicine, Hershey PA \\ ${ }^{4}$ Department of Chemistry, Department of Biomedical Engineering, Penn State University, \\ University Park, PA
}

\section{Abstract}

Thymidylate synthase (TS) is a dimeric enzyme conserved in all life forms that exhibits the allosteric feature of half-the-sites activity. Neither the reason for, nor the mechanism of this phenomenon is understood. We used a combined NMR and molecular dynamics approach to study a stable intermediate preceding hydride transfer, which is the rate limiting, and half-the-sites step. In NMR titrations with ligands leading to this intermediate, we measured chemical shifts of the apo enzyme ( $\left(\operatorname{lig}_{0}\right)$, the saturated holo-enzyme $\left(\operatorname{lig}_{2}\right)$, and the typically elusive singly bound $\left(\operatorname{lig}_{1}\right)$ states. 40 amides showed quartet patterns providing direct NMR evidence of coupling between the active site and probes greater than $30 \AA$ away in the distal subunit. Quartet peak patterns have symmetrical character, indicating reciprocity in communicating the first and second binding events to the distal protomer. Quartets include key catalytic residues and map to the dimer interface $\beta$ sheet, which also represents the shortest path between the two active sites. Simulations corroborate the coupling observed in solution in that there is excellent overlap between quartet residues and main-chain atoms having inter-subunit cross-correlated motions. Simulations identify five hotspot residues, three of which lie at the kink in the unique $\beta$-bulge abutting the active sites on either end of the sheet. Inter-strand cross-correlated motions become more organized and pronounced as the enzyme progresses from $\operatorname{lig}_{0}$ to $\operatorname{lig}_{1}$, and ultimately $\operatorname{lig}_{2}$. Coupling in the apparently symmetrical complex has implications for half-the-sites reactivity and potentially resolves the paradox of inequivalent TS active sites despite the vast majority of X-ray structures appearing symmetrical.

*Corresponding author: sapienza@email.unc.edu.

Supporting Information

Rendering of all quartet resonance patterns, differences in $S^{2}$ axis between lig0 and lig2 states mapped onto the structure and as a function of distance from diligand, and distribution of "supershifting" quartets within the structure.

Accession Codes

E. coli TS: UniProtKB P0A884 (TYSY_ECOLI)

P. carinii TS: UniProtKB - P13100 (TYSY_PNECA) 


\section{Introduction}

Liberal definitions of allostery that simply require distal elements of a protein to be affected by perturbation, suggest that most proteins can be viewed as allosteric ${ }^{1-4}$. This view is supported by a growing body of evidence demonstrating that mutation, binding, and protonation, all produce changes in structure, dynamics, or both, in regions remote from the site of modification ${ }^{4-10}$. The power of allostery is apparent when these intrinsic features are harnessed by evolution to yield exquisite regulatory mechanisms in the cell ${ }^{11}$, or by scientists to design allosteric drugs and proteins ${ }^{12-15}$. Classic definitions of allostery require multiple ligand binding events such that binding of the first ligand either promotes or inhibits binding in the second site ${ }^{16,17}$. The most canonical examples of this classical type are multimeric proteins ${ }^{18-20}$, in which multiple active sites lie within or at the interface between individual protomers. Among these, dimers are the most common oligomers ${ }^{21}$ and are highly represented in the set of allosteric proteins ${ }^{22-27}$. Dimers also tend to be the simplest to study, especially by NMR, arguably the most powerful tool for assessing both structure and dynamics of biomolecules, because of the size limitations associated with the method. Collectively, these factors make dimers attractive systems for studying the critical phenomenon of allosteric communication.

Thymidylate synthase (TS, UniProt ID: P0A884) is a dimeric enzyme responsible for synthesis of the sole de novo source of deoxythymidine monophosphate (dTMP) in most organisms. Some pathogenic microbes utilize two forms of TS, one canonical thyA-type and the other a Flavin dependent enzyme, or thyX-type ${ }^{28,29}$. In addition, although some organisms use so-called bifunctional enzymes, where TS and another enzyme in the folate metabolism cycle, dihydrofolate reductase, are encoded on the same polypeptide ${ }^{30}$, the fold and primary sequences of thyA-type TS enzymes are highly conserved in all forms studied to date. TS uses a multiple step reaction coordinate along which both a methylene and hydride are donated from the methylenetetrahydrofolate (mTHF) cofactor to the deoxyuridine monophosphate (dUMP) substrate. Because the product is a critical precursor for DNA synthesis and, therefore, required for cell division, TS has attracted attention as a drug target for treatment of anti-cancer and antimicrobial diseases. Indeed, there are several chemotherapy drugs on the market that bind to the TS active site ${ }^{31}$.

TS is also an attractive system to investigate because it is classically allosteric. The architecture of this homodimeric enzyme appears to be set-up for allostery since the active sites are comprised of residues from both subunits. Furthermore, studies of TS from several organisms show it is a half-the-sites enzyme in that only one active site performs the rate limiting 32 hydride transfer step at a time ${ }^{33,34}$. Despite the wealth of structural, kinetic, and thermodynamic literature on TS from multiple species, there is no consensus about the nature and mechanism of allostery. While the kinetic data on half-the-sites activity are compelling, there are conflicting reports about the existence and magnitudes of allostery in the substrate and cofactor binding steps. Some experiments suggest that TS has negative cooperativity in substrate or cofactor binding $32,35-38$. Yet our ITC and NMR experiments strongly support the conclusion that at least for the E. coli enzyme, both binding sites are not only competent to bind substrate and cofactor, but the intrinsic affinities associated with the 
first and second binding events for both molecules are similar, differing at most by a factor of two ${ }^{39,40}$. This observation is consistent with a number of X-ray crystal structures of bound forms of the E. coll enzyme, where both active sites are filled and both protomers are symmetrical ${ }^{41}$. TS also appears to be symmetrical in solution based on TROSY ${ }^{1} \mathrm{H}_{-}{ }^{15} \mathrm{~N}$ HSQC NMR spectra of the free, dUMP bound, and 5-fluoro deoxyuridine monophosphate (FdUMP)-mTHF bound enzymes. These spectra have only one set of resonances 40,42 indicating that any dynamic excursions away from symmetry are fast on the NMR timescale.

There are two TS crystal structures with asymmetry dramatic enough to explain half-thesites activity. The first is a model of TS from the fungus, Pneumocystis carinii ${ }^{43}$, which is $49 \%$ identical to E. coli TS and $62 \%$ identical to the human form. It should be noted that there are no kinetic or thermodynamic data on this fungal enzyme, so it is not possible to comment on the nature of allostery (i.e. cooperative binding, half-the-sites activity, or both) in this particular TS and how it relates to the structural asymmetry. The second example of asymmetry comes from an X-ray model of the single amino acid C-terminal deletion mutant of E. coli TS (I264Am) in complex with dUMP and CB3717 ${ }^{44}$. The authors hypothesize that this asymmetry may also exist in the wild-type enzyme, albeit in a much subtler, yet still functionally relevant form ${ }^{44}$. Nonetheless, the asymmetries observed in the crystal lattices of Pneumocystis carinii TS and E. coli I264Am TS, if they exist in solution, are outliers in ternary complexes. The dUMP/CB3717 complexes are perfectly symmetrical in crystal structures of E. coli TS ${ }^{45}$, and another highly studied TS from Lactobacillus casei (PDBID 1LCA). Likewise, a crystal structure of the human enzyme with dUMP and a similar cofactor analog, Raltitrexed, has slight differences between the subunits (all atom root-mean square distance $(\mathrm{RMSD})=0.17 \AA$ ), but both active sites are loaded ${ }^{46}$. Because hydride transfer occurs within the ternary complex with a C-terminus that is closed over an active site with substrate and cofactor bound, and because ternary complexes in most forms of TS are symmetrical in structural models of this state, we operate under the assumption that halfthe-sites reactivity must be understood in terms of dynamic excursions away from a (nearly) symmetrical form.

As a means to assess dynamic communication between active sites in E. coli TS, we used a combined NMR and molecular dynamics approach. We took advantage of favorable NMR characteristics of spectra involving substrate analog, FdUMP, and the biological cofactor, mTHF. We collectively refer to these molecules as the "diligand" 42 because C6 of the FdUMP moiety is covalently bonded to Cys 146 of the enzyme, and C5 of FdUMP is covalently bonded to mTHF via a methylene bridge. Diligand is, therefore, viewed as a mechanistic inhibitor ${ }^{47}$ that halts the reaction after methylene bridge formation, but prior to resolving methylene transfer, and prior to hydride transfer. This trapped intermediate is, therefore, the most stable form that allows us to study features of the complex preceding the half-the-sites hydride transfer step. We are able to measure the chemical shifts of the apo $\left(\operatorname{lig}_{0}\right)$, saturated ( $\left.\operatorname{lig}_{2}\right)$, and the typically elusive and information rich singly bound states $\left(\operatorname{lig}_{1}\right)$ in diligand titrations. In some cases, the singly bound state yielded distinct resonances for the bound and unbound protomers, giving rise to quartet resonance patterns. Previously, we utilized these quartets to quantify the fractions of each of the $\operatorname{lig}_{0}, \operatorname{lig}_{1}$, and $\operatorname{lig}_{2}$ species as a means to measure the relative affinities of the first and second binding sites ${ }^{40}$. Here, we interrogate the quartets as a means to understand inter-subunit communication. We found 
that some elements of the distal subunit are insulated from the local binding event, but quartet residues highlighted elements of the distal subunit that are coupled to binding. Quartets also suggest that coupling is bidirectional in that binding of the first ligand affects the distal subunit and binding of the second affects the structure and/or dynamics of the prebound site. Furthermore, by combining information gleaned from NMR and molecular dynamics simulations of the $\operatorname{lig}_{0}, \operatorname{lig}_{1}$, and $\operatorname{lig}_{2}$ states, we are able to show that the two active sites in TS communicate with one another by using the intervening $\beta$-sheet that also forms the dimer interface. Simulations reveal that active sites have minimal communication in the $\operatorname{lig}_{0}$ state, but rather a network of correlated motions involving the two active sites is triggered by the first diligand binding event and amplified upon binding the second. Contacts between the diligand and a unique right handed $\beta$-bulge feature of this sheet are likely the triggering events. The fact that the inter-active site correlated motions are most pronounced in the symmetrical $\operatorname{lig}_{2}$ state could provide an explanation of half-the-sites reactivity in TS and resolve the paradox of allostery despite the appearance of symmetry in static crystal structures.

\section{Materials and Methods}

\section{Materials, enzyme expression, and enzyme purification}

$\mathrm{U}-\left[{ }^{2} \mathrm{H},{ }^{15} \mathrm{~N}\right] \mathrm{TS}$ was expressed, purified, and exchangeable deuterium atoms were "backexchanged" to protons as described previously ${ }^{48}$. 5-Fluoro- $2^{\prime}$-deoxyuridine $5^{\prime}$ monophosphate (FdUMP) was purchased from Sigma and (6R)-5,10methylenetetrahydrofolate (mTHF) was purchased from Merck \& Cie (Switzerland).

\section{NMR resonance assignments}

Amide chemical shift assignments for the $\operatorname{lig}_{0} 42$ and diligand $\operatorname{lig}_{2} 48$ states were reported previously. Diligand $\operatorname{lig}_{1}$ resonances were assigned based on proximity to previously assigned $\operatorname{lig}_{0}$ and $\operatorname{lig}_{2}$ peaks. We took care to add equivalent amounts of FdUMP and mTHF in titrations and all other NMR experiments. This ensures pure states from the diligand binding manifold and no contamination by the FdUMP only, or mTHF only manifolds. Accurate assignment of $\operatorname{lig}_{1}$ resonances to either the empty or bound subunit were made possible by use of mixed labeled dimer in which a ${ }^{15} \mathrm{~N}$-labeled wild-type subunit was paired with an unlabeled R126E, R127E mutant ${ }^{42}$. Such a dimer is only competent to bind a single diligand molecule ${ }^{49}$, therefore giving rise to an HSQC spectrum of the $\operatorname{lig}_{1}$ empty subunit. It is counterintuitive that the wild-type subunit $\left({ }^{15} \mathrm{~N}\right.$ labeled in this case) is empty, but it is the case because the active site is comprised of residues from both subunits and R126 and R127 from the wild-type subunit complement the remaining active site residues in the mutant subunit to form the single canonical active site. We were able to assign resonances to all four microstates for 207 out of 250 non-proline amides. We have $\operatorname{lig}_{0}$ and $\operatorname{lig}_{2}$ assignments for many of the remaining resonances, but half saturated diligand spectra are too crowded in places to be certain of singly bound resonances.

\section{NMR chemical shift perturbation spin relaxation}

Chemical shift perturbation based on TROSY ${ }^{1} \mathrm{H}-{ }^{15} \mathrm{~N}$ HSQC NMR spectra were calculated using the following: 


$$
C S P=\sqrt{\Delta \delta_{H N}^{2}+0.1 * \Delta \delta_{N}^{2}}
$$

Chemical shift projection analysis (CHESPA) was carried out as described ${ }^{50,51}$ with the A and $B$ vectors defined by scheme 1 below: and the two reported parameters in Figure 4: the fractional shift $(\mathrm{X})$ and $\cos (\theta)$, are the projection of vector $\mathrm{A}$ on vector $\mathrm{B}$, and the cosine of the angle between vectors $A$ and $B$ respectively ${ }^{50}$. Note that the red $\operatorname{lig}_{1}$ resonance, which is associated with the bound subunit in the single bound state is fully schematized above. However, we calculate the fractional shift and angular values from the empty subunit lig 1 resonance as well (shown above in green to complete a quartet).

Methyl NMR order parameters were measured using the triple quantum relaxation violated coherence transfer approach developed by Kay and coworkers 52 and applied to TS as described previously ${ }^{39}$. The global tumbling times, $\boldsymbol{\tau}_{\mathrm{m}}$, which are required for calculation of methyl symmetry axis order parameters $\left(S_{\text {axis }}^{2}\right)$, were measured previously for the apo enzyme ${ }^{39}$ and diligand saturated, lig 2 complex ${ }^{45}$ based on TROSY versions of ${ }^{15} \mathrm{~N} R_{1}$, $R_{1} \rho$, and $\left\{{ }^{1} \mathrm{H}\right\}-{ }^{15} \mathrm{~N}$ heteronuclear NOE pulse sequences ${ }^{53}$.

\section{Molecular dynamics simulations}

For the two symmetrical states, the $\mathrm{lig}_{0}$ and $\mathrm{lig}_{2}$, high resolution $\mathrm{X}$-ray models (PDB ID: 2FTQ for $\operatorname{lig}_{0}$ and PDB ID: 1 TSN for $\left.\operatorname{lig}_{2}\right){ }^{47}$ were used as starting structures during MD simulations. For the $\operatorname{lig}_{1}$ simulation, the diligand was simply removed from one of the active sites of $\operatorname{lig}_{2}$ (PDB ID: 1TSN) prior to simulation. All starting structures were optimized and all hydrogen were reconstructed using Maestro Protein and Ligand preparation tools (Schrödinger, LLC). All MD simulations were performed using GROMACS 2018 package 54, 55 using CHARMM36 force field ${ }^{56}$. The diligand was parametrized using the official CHARMM General Force Field server (CGenFF) ${ }^{57,58}$. All MD simulations were at a constant pressure and temperature of $1 \mathrm{~atm}$ and $298 \mathrm{~K}$, respectively. Particle mesh Ewald (PME) was used for long-range electrostatic interactions with $10-\AA$ cutoff for non-bonded interactions. Each system was solvated using the TIP3P water model and neutralized with sodium and chloride ions. Systems were further equilibrated using NVT and NPT thermostats. During these equilibrations the diligands were constrained to their initial positions with harmonic constraints. Prior to production simulations, we ran a series of short simulations during which we were closely monitoring the ligand positions in the binding site to ensure that key distances between the ligands and the protein atoms correspond to ones of the X-ray structures. Next, we performed productive simulations for $500 \mathrm{~ns}$ for each system $\operatorname{lig}_{0}, \operatorname{lig}_{1}$ and $\operatorname{lig}_{2}$. Based on the analysis of the root-mean-square deviation (RMSD) of backbone $\mathrm{C}^{\mathrm{a}}$ positions, the first $50 \mathrm{~ns}$ of the simulations were omitted for further analysis to avoid initial system's fluctuations due to equilibration. The structures and representative snapshots of the trajectories were visually inspected using PyMol 2.1 (Schrödinger, LLC).

\section{Cross-correlation analysis}

Each trajectory then was divided into three pieces (100-200ns 250-350ns, 400-500ns) that were used to calculate cross-correlation motion correlation matrices ${ }^{12,59}$. To remove the 
noise, we performed following filtering procedure. For each of the cross-correlation matrices, we plotted a distribution of cross-correlation coefficient values. Based on the distributions we kept only values corresponding to the top $90 \%$ strongest correlations, both negative (anti correlations) and positive (correlations). Because the distributions are close to normal this procedure is equivalent to keeping the values in the tails of distributions with the absolute value of Z-score greater than 1.65 .

\section{Results and Discussion}

\section{Ligand state peak multiplets in TS diligand titrations: Doublet peak patterns reveal regions of subunit independence.}

TROSY ${ }^{1} \mathrm{H}^{15}{ }^{15} \mathrm{~N}$ HSQC spectra of apo ${ }^{40}$ and diligand-saturated ${ }^{48}$ TS exhibit single sets of resonances (Figure 1A), which indicates both states are symmetrical homodimers in solution just as they are in the crystal lattice ${ }^{47,60}$. Many of the apo resonances shift upon diligand binding (Figure 1A), consistent with the significant conformational change that occurs in going from the open conformation of the free enzyme, to the closed ternary complex, in which the C-terminus clamps down on the reactants and shields them from solvent ${ }^{41}$. Previously, we showed resonances in diligand titrations ${ }^{40}$ are in slow exchange on the NMR timescale, allowing us to monitor the populations and chemical shifts of all possible micro states in the ensemble comprised of: apo enzyme $\left(\operatorname{lig}_{0}\right)$, doubly bound enzyme $\left(\operatorname{lig}_{2}\right)$, and the singly bound enzyme, $\operatorname{lig}_{1}$, which, in principle may have subunit specific signals from the bound $\left(\operatorname{lig}_{1 \mathrm{~B}}\right)$ and empty $\left(\operatorname{lig}_{1 \mathrm{E}}\right)$ protomers. We referred to these patterns as "ligand state peak multiplets" ${ }^{42}$. We were able to assign resonances to all four microstates for 207 out of 250 non-proline residues in TS, and the most common peak multiplet pattern at intermediate titration points is the doublet, which we observe for 121 out of 207 amides. A doublet is derived from two sets of overlapping signals; one from the $\operatorname{lig}_{0}$ and $\operatorname{lig}_{1 \mathrm{E}}$ states, and the other from the $\operatorname{lig}_{2}$ and $\operatorname{lig}_{1 \mathrm{~B}}$ states (Figure 1B). This observation suggests that for a large number of amides, the chemical environments of the free and bound subunits in the $\operatorname{lig}_{1}$ state are the same as in the symmetrical $\operatorname{lig}_{0}$ and $\operatorname{lig}_{2}$ species, respectively. Mapping the doublet amides onto the structure of TS reveals which regions of the distal subunit that are insulated from the effects of diligand binding to the local subunit (Figure 1C). These insulated regions are wide-spread throughout the structure. Furthermore, because the doublets coincide with the C-terminus and other regions that undergo the largest conformational changes (Figure 1C), binding of the first diligand does not elicit a concerted conformational change in the second binding site. This finding is not surprising for TS since this conformational change closes off the binding site, ensuring it must happen after diligand binding.

\section{Ligand state peak multiplets in TS diligand titrations: Quartet patterns show coupling of both binding events to distal subunits.}

Some parts of the distal subunits sense individual diligand binding events as evidenced by the 40 plus amides showing quartet peak multiplet patterns (Figure 2A). The existence of this pattern provides direct, solution-based evidence of coupling between the active site and remote elements of the enzyme. Quartet amides in the distal subunit lie as far as $35 \AA$ from the nearest diligand atom in the bound subunit, highlighting long distance communication of the binding event. The distal effects are not simple manifestations of proximity to the 
diligand or conformational change in the local subunit since the distribution of quartet residues do not radiate in a spherical pattern centered on the diligand, nor do they track with conformational change in the local subunit (Figure 2A). Rather, the quartet amides cluster to the $\beta$-sheet that forms the TS dimer interface and connects the two active sites. It is important to emphasize that a quartet pattern signifies both subunits of the singly bound state have chemical shifts that are distinct from the two symmetrical $\operatorname{lig}_{0}$ and $\operatorname{lig}_{2}$ states. It follows that for these amides, there are two local and two distal responses commensurate with the two binding events (Figure 2B). The local effects are simply the amide chemical shift perturbations (CSPs) in the first and second subunits upon binding the first and second diligand molecules, respectively. We denote the magnitudes of these effects as local 1 and local $_{2}$. The first distal effect, distal 1 , reports on the perturbation to the empty subunit in response to binding the first diligand, and the second distal effect, $\operatorname{distal}_{2}$, measures the effect on the pre-bound subunit in response to the $\operatorname{lig}_{1}$ to $\operatorname{lig}_{2}$ transition (Figure 2B).

One of the striking features of the quartet patterns is their symmetrical appearance (Figure $2 \mathrm{C}$ and Figure $\mathrm{S} 1$ ). In most cases the symmetry is not perfect, in the sense that the magnitudes of the local CSPs tend to be greater than those within the distal subunit (Figure 2D). This must be further qualified because the TS active site is comprised of residues from both subunits and in some cases the amino acids that are closest to the local binding event lie within the "distal" subunit (e.g. Arg127 in Figure 2D). Nonetheless, quartet lig 1 resonance positions tend to be symmetrically apposed with respect to the axis that connects the $\operatorname{lig}_{0}$ and $\operatorname{lig}_{2}$ resonances (Figure 2C). This type of behavior suggests TS adheres to the allosteric principle of reciprocity 61,62 in which the effects of a perturbation on distal sites are bidirectional. This means that for the case of a two-ligand-binding dimer, the effect of the first binding event on a distal site is similar to the effect of the second diligand binding event on the reciprocal site in the pre-bound subunit. To quantify reciprocity, the ratios of the two local CSPs and the two distal CSPs (Figure 2D) are plotted in Figure 2E. A value of 1.0 for these ratios represents perfect reciprocity. Indeed, the two ratios for the quartet residues cluster around 1.0. There are rare exceptions to this high degree of reciprocity (e.g. Ile29, Asp124, Arg127, Val135, and Gly204 in Figure 2E) but the quartet geometrical patterns for these residues are still highly symmetrical as defined above (Figure S1). On one hand reciprocity should be expected a priori in this system given it is a symmetric homodimer and the fact that the dimer interface $\beta$-sheet, which contains most of the quartet residues, does not undergo a conformational change upon binding diligand ( $\beta$-sheet RMSD $=0.27 \AA$, Figure $3 \mathrm{~A}$ ). On the other hand, there are several reasons to expect deviations from reciprocity as read out by the chemical shift, even in the case of this (or any) symmetric dimer ${ }^{63}$. First, and most trivially, but nonetheless critical to consider, the presence of the diligand itself affects the electronic environment of nearby amides and induces asymmetry in the system. Second, binding of the first diligand does induce a conformational change in the local binding site and the majority of the distal subunit is insulated from this change (see interpretation of doublet patterns above), thus the "medium" in which the allosteric signal is transmitted, while symmetrical in amino acid composition, does have differences in structure and/or dynamics as sensed by the first and second binding events. In further support of this idea are NMR relaxation experiments in which we compared the ps-ns dynamics of Ile, Leu, and Val methyl probes in the $\operatorname{lig}_{0}$ and diligand $\operatorname{lig}_{2}$ states. These experiments show that 
diligand binding affects methyl dynamics throughout the protein (Figure S2), including multiple residues within the $\beta$-sheet (Figure 3B). We note that the methyl resonances of these probes are not sufficiently resolved in the singly bound state to allow quantification of dynamics in the $\operatorname{lig}_{1}$ protomers, but differences among the two symmetrical end states are highly suggestive of dynamic changes in $\operatorname{lig}_{1}$, and support the idea of changes in the allosteric signal transmission "medium" put forth above. Taken together, while the structure of the sheet is unchanged, the first diligand binding event certainly induces structural asymmetry and likely causes dynamic inequality of the subunits that could in principle make the local ${ }_{1}$ and local ${ }_{2}$ CSPs different, and/or result in differences between distal ${ }_{1}$ and distal $_{2}$. However, the observed reciprocity suggests that either the intrinsic symmetry of the dimer is dominant, there was evolutionary pressure to ensure bidirectional communication, or both.

\section{Ligand state peak multiplets in TS diligand titrations: CHESPA points to unique role of the $\beta$-sheet kink.}

We examined the quartets for patterns (aside from the pseudo symmetry described above) that could explain the structural and dynamic origins of the interesting chemical shift phenomena. Chemical shift projection analysis (CHESPA) is a tool for identifying residues in allosteric networks involving conformational transitions that are fast on the NMR timescale $50,51,64$. The tool assesses the magnitude and direction of a peak shift associated with some query state relative to some reference state as compared to the shift in going from the same initial reference state to a final state (Scheme 1 in Methods). In the case of a single amide reporting on diligand binding, the reference initial state is a $\operatorname{lig}_{0}$ resonance, the final state is a $\operatorname{lig}_{2}$ resonance, and the query state is a $\operatorname{lig}_{1}$ peak, coming from either the empty or bound protomer. CHESPA gives two different metrics for each quartet: The first is called the fractional shift $(\mathrm{X})$, which is a measure of the relative shift of the query peak as compared to the magnitude of the vector connecting the $\operatorname{lig}_{0}$ and $\operatorname{lig}_{2}$ resonances, and the second, $\cos (\theta)$, reports on the orientation of the query vector connecting $\operatorname{lig}_{0}$ to $\operatorname{lig}_{1}$ relative to the reference vector connecting $\operatorname{lig}_{0}$ and $\operatorname{lig}_{2}$ resonances (Scheme 1, methods). In general, the fractional shift values for $\operatorname{lig}_{1}$ resonances in the bound subunit are positive and $\cos (\theta)$ values are close to one (Figure 4) suggesting these peaks shift toward and are collinear with the $\operatorname{lig}_{2}$ resonance. The same pattern exists for the majority of $\operatorname{lig}_{1}$ peaks from the empty subunit, indicating regions with quartets become more like the saturated diligand complex in the singly bound state (Figure 4). However, CHESPA illuminates more subtle features of quartet patterns. If binding of the first diligand molecule caused a global shift in populations of some pre-existing two-state conformational exchange process in either subunit, the $\cos (\theta)$ values would cluster around 1.0 and the fractional shifts would all be of the same sign and the same magnitude. This is not what we observe. Both the magnitudes and directions of the $\operatorname{lig}_{1}$ vectors are variable indicating more complex behavior. As a dramatic example, a group of eight resonances have fractional shift values greater than 1.0 in the bound subunit, indicating that the $\operatorname{lig}_{1}$ resonance shifts beyond the $\operatorname{lig}_{2}$ peak. We coined this phenomenon as "supershifting" based on previous observations with binding of substrate (dUMP) to TS ${ }^{42}$. Another hallmark of the eight diligand supershifters is the $\operatorname{lig}_{1}$ resonance in the empty subunit shift away from the $\operatorname{lig}_{2}$ resonance as indicated by negative fractional shifts and negative $\cos (\theta)$ (Figure 4). This pattern is illustrated by multiple examples of supershifting quartets in NMR spectra in Figure 2B (Gly203) and Figure 2C (Leu119, Gln165, and 
Gly203). These supershifting residues cluster to two regions of TS: Five of the eight residues $(32,165,166,203$, and 204) map to the dimer interface $\beta$-sheet kink, and the remainder $(119,178$, and 194) reside in helices that are adjacent to the sheet but have side chains that pack against it (Figure S3). The importance of the $\beta$-sheet kink in inter-active site communication is discussed in greater detail below in the context of molecular dynamics simulations. Taken together, CHESPA analysis of the quartets suggests that while all the patterns share the feature of pseudo-symmetry, they do not reflect a global shift in a single, two-state conformational equilibrium; rather, multiple processes must be at play.

\section{Ligand state peak multiplets in TS diligand titrations: Direct evidence of coupling between the two active sites.}

Chemical shifts of the $\operatorname{lig}_{1}$ state show that binding events are communicated to critical active site residues separated by greater than $30 \AA$. For example, Cys146 provides the $\mathrm{S}^{-}$ nucleophile that attacks the C6 position of substrate dUMP ${ }^{65}$ and also lends binding stability to the binary complex ${ }^{39}$ (Figure 5A). At intermediate diligand concentrations, amide resonances for Cys146 are split near the position of the $\operatorname{lig}_{2}$ peak (Figure 5B), suggesting this residue in the bound subunit is in a different environment depending on whether the distal active site is free or occupied. We are unable to conclusively state that Cys 146 yields a quartet since the peak in the $\operatorname{lig}_{0}$ state is broadened away ${ }^{39}$, but the distinct resonances of $\operatorname{lig}_{1 \mathrm{~B}}$ and $\operatorname{lig}_{2}$ is highly suggestive of bidirectional communication given the near universal reciprocity noted above. The same logic applies to the behavior of $\operatorname{Arg} 21$, one of the three amino acids making charged interactions to the dUMP phosphate. This sidechain also stabilizes the closed conformation of the $\mathrm{C}$-terminus by making a hydrogen bond with the C-terminal carboxylate group (Figure 5A) and therefore is important in both binding and catalysis. The amide resonance for Arg21 is also broadened away in the $\operatorname{lig}_{0}$ state but there is clear doubling of the peak near the $\operatorname{lig}_{2}$ resonance (Figure 5B). Interestingly, in the two examples of TS X-ray models with asymmetric binding sites (ternary complexes of the E. coli C-terminal deletion mutant and wild-type Pneumocystis carinii), Cys146 and Arg21 (or the homologous residues in the case of the Pneumocystis carinii structure) are among the residues with notable differences in the two binding sites 43,44. As a final example, Arg 166 gives a bona fide quartet pattern (Figure 5B). Arg 166 is implicated in binding by virtue of making electrostatic contacts to the dUMP phosphate (Figure 5A), and it has been shown to also play critical roles in chemical steps by stabilizing the nucleophilic form of Cys146 and stabilizing the transition state for hydride transfer ${ }^{66}$. Collectively, these data directly show coupling between the two active sites.

\section{Inter subunit cross-correlated motion hotspots localize to the dimer interface and specifically the $\beta$-bulge kink}

While the NMR quartets demonstrate that binding events are communicated between the active sites, the chemical shifts are not sufficient to reveal mechanism. For mechanistic insight, we turned to molecular dynamics (MD) simulations: $500 \mathrm{~ns}$ simulations of the $\operatorname{lig}_{0}$, $\operatorname{lig}_{1}$, and $\operatorname{lig}_{2}$ states were performed (Methods). For the two symmetrical states, the $\operatorname{lig}_{0}$ and $\operatorname{lig}_{2} \mathrm{X}$-ray models, 2FTQ and 1TSN, respectively, were used as starting coordinates, and for the $\operatorname{lig}_{1}$ simulation, the diligand was removed from one of the active sites prior to simulation. To assess communication we calculated the $\mathrm{C}^{\mathrm{a}}$ atom cross-correlated motion matrices 
12,5967. Only strong correlation values were kept, where the thresholds were chosen based on the cross-correlation values distribution ${ }^{59}$ (Methods). The filtered inter subunit crosscorrelated motion maps for the three states are shown in Figure 6. There is clearly an increase in the extent and organization of cross-correlations as TS progresses from lig $_{0}$ to $\operatorname{lig}_{1}$, and finally to the $\operatorname{lig}_{2}$ state, yet a single diligand binding event is sufficient to recapitulate some of the correlated motions seen in the saturated ensemble (Figure 6). The $\operatorname{lig}_{1}$ inter subunit cross-correlated motion heat map is asymmetrical however, with row vectors representing how motions of the $\mathrm{C}^{\mathrm{a}}$ atom from a single residue in the bound subunit is correlated to the $\mathrm{C}^{\mathrm{a}}$ atoms of each residue in the unbound subunit. Similarly, column vectors extract correlations between single residues of the empty subunit and each residue of the bound protomer (Figure 6). In lig $_{2}$ simulations, five "hotspot" residues, Phe30, Ile129, Phe152, Arg166, and Gly204 stand out as having wide-spread and symmetrical inter-subunit cross-correlated motions (Figure 6). These correlations are limited in the $\operatorname{lig}_{0}$ ensemble, become more pronounced yet asymmetric in the lig $_{1}$ complex, and are strong and highly symmetrical in the $\operatorname{lig}_{2}$ simulations. Importantly the motions of these hotspot amino acids are correlated with motions of residues within the dimer interface $\beta$-sheet of the opposite subunit (Figure 6), yielding excellent convergence with NMR data showing quartet resonance patterns for these same residues (Compare Figure 6 with Figure 2A). Collectively these data show: (1) that the two active sites communicate with one another through the $\beta$ sheet that connects them, (2) binding of a single diligand is required to activate this communication network since it is absent in the $\operatorname{lig}_{0}$ ensemble, and (3) the network persists in an amplified and more symmetrical form in the $\operatorname{lig}_{2}$ state. Two pieces of data put limits on the timescale of the coupled motions of the active sites in the lig 2 state. First, only one set of resonances is observed in diligand saturated spectra indicating the motions are fast on the NMR timescale, which depending on the chemical shift difference between the states is on the order of $\mu$ s-ms or faster. Second, previous NMR measurements of chemical exchange did not reveal $\beta$-sheet motions on this timescale in the $\operatorname{lig}_{2}$ complex ${ }^{39}$, indicating the correlated motions must occur on the sub-microsecond timescale. Taken together, the agreement between the NMR data and diligand simulations noted above suggests that symmetric quartet resonance patterns are predictive of inter-subunit correlated motions, and leads to the hypothesis that the quartets capture rapid timescale structural excursions of the $\beta$-sheet.

One remarkable aspect of the correlated motion hotspots is that three out of five lie precisely at the vertex of the kinked dimer interface $\beta$-sheet (Figure 7A). In TS, the dimer interface is comprised of two stacked six stranded $\beta$-sheets. Each can be further subdivided into two sheets, approximately orthogonal to one another ${ }^{68}$. This right handed $\sim 90^{\circ}$ rotation, or the kink, results from a stacked $\beta$-bulge, whose stability is derived from five inter-strand hydrogen bonds between Cys146 and Gln165, Gln165 and Gly203, Arg166 and Gly204, Gly203 and His32, and Gly204 and Phe30 (Figure 7B). The bulge is in an identical conformation stabilized by the exact same hydrogen bonds in the lig $_{0}$ state (Figure 3A), yet there are few cross-correlated motions between the bulge and the opposite sheet, or between the two bulges in opposite protomers (i.e. the two active sites) (Figure 6). Why is the dynamic network activated by diligand binding? An attractive hypothesis is that the effects of contacts between bulge residues and diligand, specifically the contacts between Cys146 and FdUMP, and the electrostatic interaction between Arg166 and a phosphoryl oxygen of 
FdUMP (Figure 7B), are propagated across the dimer interface and down the sheet to the opposite active site in a bidirectional manner.

It is now appreciated that long range, motional coupling is a fundamental property of antiparallel $\beta$-sheets ${ }^{69}$. This unique feature is imparted by the relatively large area of permissible $\phi / \psi$ angles in the $\beta$-region of Ramachandran space, which allows the constituent dihedral angles to fluctuate so long as the hydrogen bonding patterns stabilizing the sheet are conserved. In this way, correlation of dihedral angles $\psi_{i-1}$ and $\phi_{i}$, propagate fluctuations in a direction parallel to the strand via so-called crankshaft motions ${ }^{70,71}$ and concerted crankshaft motions between neighboring strands propagate dynamics in a direction perpendicular to the strands via $\beta$-lever motions ${ }^{69,72}$. This explains propagation throughout the $\beta$-sheet in the local subunit, but chemical shift and MD data show that binding is coupled to the $\beta$-sheet in the opposite protomer. How does this occur? The remaining two hotspot residues, Ile129 and Phe152, likely provide insight in that they are directly across from the $\beta$-bulge hotspot in the opposite protomer. Ile129 of chainB packs against Arg166 of chain A, effectively coupling hotspots within the strand containing the binding site with those in the other subunit (Figure 7C). Interestingly, Ile129 becomes more flexible upon diligand binding (Figure 3B), consistent with involvement of this residue in any dynamic propagation mechanism. The last hotspot residue, Phe152 also packs against the main chain of bulge residue Phe30 in the opposite strand (Figure 7C). Therefore, we hypothesize that effects of binding are transmitted down the local $\beta$-beta sheet through fluctuations that conserve hydrogen bonding between strands, and across the dimer interface by maintaining the tertiary interactions between the sheets in the two subunits. Collectively, these $\beta$-sheet couplings give rise to bending and twisting of the sheet ${ }^{69,73}$ with the degree and balance between bending and twisting forms of flexibility being idiosyncratic to the fold. In the case of TS, X-ray models do not show any change in $\beta$-sheet structure in going from $\operatorname{lig}_{0}$ to $\operatorname{lig}_{2}$ (Figure 3A), so differences in these couplings are likely not sufficient to cause large scale structural deformities, but they are strong enough to manifest in chemical shift changes, changes in dynamic coupling, and perhaps differences in activity between the two coupled active sites.

\section{A revised mechanism for allosteric coupling in TS.}

The two previously described X-ray structures of TS that could give insight into half-thesites activity are characterized as having significant asymmetry between the two active sites. In the case of a Pneumocystis carinii TS ternary complex ${ }^{43}$, one site has substrate and cofactor analog bound, and the other is loaded with only substrate. The non-functional site showed no evidence of the typical covalent bond between the active site cysteine and substrate, and the C-terminus was not closed, both of which are required for hydride transfer. A difference distance matrix comparison of the coordinates from the two subunits identified two continuous segments that could potentially transmit the signal from one active site to the other: The first, Cys173-Phe176 (homologous to E. coli TS Cys146-Phe149) is at the edge of the dimer interface $\beta$-sheet and contains the active site cysteine, and the second, Leu196Arg199 (E. coli Leu163-Arg166) lies within the adjacent strand and includes a $\beta$-bulge hotspot residue (E. coli $\operatorname{Arg} 166$ ) as identified in the MD simulations described above. We see corroborating evidence in this work in that these same residues have quartet patterns in 
E. coli diligand titrations and significant inter-subunit cross-correlated motions in simulations. Despite this agreement, X-ray models of E. coli ternary complexes are symmetrical and both sites appear identically poised for hydride transfer. Why are the ternary complex structures of these two homologous enzymes dramatically different? We propose three possibilities: (1) The crystal lattice may impose asymmetry in the Pneumocystis carinii lattice that does not exist in solution, (2) Pneumocystis carinii is a eukaryote with a TS enzyme that is $45 \%$ identical to that of $E$. coli, leaving open the possibility for different functional behavior, and (3) somewhat related to point (2) above, Pneumocystis carinii TS has three insertions, ranging from 6-12 amino acids compared to $E$. coli, and one of these, Ser92-Lys103, is very close to the C-terminus, which could differentially affect cofactor binding in the enzymes from the two species. Solution experiments are underway to interrogate active site communication in TS enzymes from higher organisms in order to differentiate from among the above hypotheses.

The second case of asymmetry was observed in a crystal structure of a single residue, Cterminal truncation of the E. coli enzyme, I264Am ${ }^{44}$. In this ternary complex, both active sites were filled with the two co-substrates, however the active site geometries and poses of the co-substrates were only consistent with catalysis in one of the sites. Again, there is some agreement between residues that differ among the two protomers of the X-ray model and quartet residues identified by NMR in this work, including Arg21, Cys146, and Arg 127. However, in general, the dimer interface $\beta$-sheet of I264Am is two-fold symmetric despite the overall asymmetry of the active sites ${ }^{44}$. This structure differs from the crystal structure of Pneumocystis carinii described above, but agrees with NMR spectra of the wild-type ternary complex (single resonances for $\beta$-sheet residues), which supports the hypothesis that TS enzymes from different organisms may have different degrees and/or mechanisms of subunit communication. Can we reconcile the asymmetric complex observed in the I264Am mutant with the single sets of resonances we see in wild-type ternary complex? The NMR data of the wild-type enzyme necessitate a mechanism that gives time averaged symmetry, yet permits instantaneous asymmetry of the two active sites. The mechanism based on the I264Am structure predicts opening and closing of the C-terminus and significant dynamics of the co-substrates in the binding pocket within the $\operatorname{lig}_{2}$ ensemble. We previously reported NMR backbone order parameters which show only slightly increased picosecondnanosecond dynamics of the C-terminus $\left(S^{2} \approx 0.7\right)$ relative to elements of secondary structure in the same complex $\left(S^{2} \approx 0.8\right){ }^{45}$. This effect is very subtle and may not be sufficient to produce the large amplitude motions predicted by the structural and kinetic ${ }^{74}$ data of I264Am. Furthermore, we did not detect $\mu \mathrm{s}$-ms motions of the C-terminus based on HahnEcho ${ }^{15} \mathrm{~N} R_{2}$ measurements of the $\operatorname{lig}_{2}$ diligand complex ${ }^{39}$. These observations suggest caution in extrapolating the specifics of inter-active site communication from the crystal structure of a mutant to the wild-type enzyme in solution. Indeed, the authors suggested that the structural excursions that make one site catalytically competent need not be as dramatic as was observed in the I264Am crystal structure. Our model, based on the aggregate X-ray, MD, and NMR data suggest that half-the-sties activity is manifest by instantaneous asymmetry in the context of time averaged symmetry. Additional NMR and MD experiments are underway in order to elucidate the exact nature of these excursions away from the average structure. 


\section{Supplementary Material}

Refer to Web version on PubMed Central for supplementary material.

\section{Acknowledgments}

This work was funded by NIH grant GM083059 (to A.L.L.) and R01-GM123247 (to N.V.D.). We thank Jeffery Bonin for helpful discussions. Experiments at the UNC School of Medicine NMR facility were supported in part by the National Cancer Institute of the National Institutes of Health under award number P30CA016086. The content is solely the responsibility of the authors and does not necessarily represent the official views of the National Institutes of Health.

\section{References}

[1]. Wodak SJ, Paci E, Dokholyan NV, Berezovsky IN, Horovitz A, Li J, Hilser VJ, Bahar I, Karanicolas J, Stock G, Hamm P, Stote RH, Eberhardt J, Chebaro Y, Dejaegere A, Cecchini M, Changeux JP, Bolhuis PG, Vreede J, Faccioli P, Orioli S, Ravasio R, Yan L, Brito C, Wyart M, Gkeka P, Rivalta I, Palermo G, McCammon JA, Panecka-Hofman J, Wade RC, Di Pizio A, Niv MY, Nussinov R, Tsai CJ, Jang H, Padhorny D, Kozakov D, and McLeish T (2019) Allostery in Its Many Disguises: From Theory to Applications, Structure 27, 566-578. [PubMed: 30744993]

[2]. Gunasekaran K, Ma B, and Nussinov R (2004) Is allostery an intrinsic property of all dynamic proteins?, Proteins 57, 433-443. [PubMed: 15382234]

[3]. Dokholyan NV (2016) Controlling Allosteric Networks in Proteins, Chemical reviews 116, 64636487. [PubMed: 26894745]

[4]. Clarkson MW, and Lee AL (2004) Long-range dynamic effects of point mutations propagate through side chains in the serine protease inhibitor eglin c, Biochemistry 43, 12448-12458. [PubMed: 15449934]

[5]. Petit CM, Zhang J, Sapienza PJ, Fuentes EJ, and Lee AL (2009) Hidden dynamic allostery in a PDZ domain, Proc Natl Acad Sci U S A 106, 18249-18254. [PubMed: 19828436]

[6]. Mauldin RV, and Lee AL (2010) Nuclear magnetic resonance study of the role of M42 in the solution dynamics of Escherichia coli dihydrofolate reductase, Biochemistry 49, 1606-1615. [PubMed: 20073522]

[7]. Clarkson MW, Gilmore SA, Edgell MH, and Lee AL (2006) Dynamic coupling and allosteric behavior in a nonallosteric protein, Biochemistry 45, 7693-7699. [PubMed: 16784220]

[8]. Fuentes EJ, Der CJ, and Lee AL (2004) Ligand-dependent dynamics and intramolecular signaling in a PDZ domain, Journal of molecular biology 335, 1105-1115. [PubMed: 14698303]

[9]. Moorman VR, Valentine KG, Bedard S, Kasinath V, Dogan J, Love FM, and Wand AJ (2014) Dynamic and thermodynamic response of the Ras protein Cdc42Hs upon association with the effector domain of PAK3, Journal of molecular biology 426, 3520-3538. [PubMed: 25109462]

[10]. Williams JK, Zhang Y, Schmidt-Rohr K, and Hong M (2013) pH-dependent conformation, dynamics, and aromatic interaction of the gating tryptophan residue of the influenza M2 proton channel from solid-state NMR, Biophys J 104, 1698-1708. [PubMed: 23601317]

[11]. Monod J, Changeux JP, and Jacob F (1963) Allosteric proteins and cellular control systems, Journal of molecular biology 6, 306-329. [PubMed: 13936070]

[12]. Karginov AV, Ding F, Kota P, Dokholyan NV, and Hahn KM (2010) Engineered allosteric activation of kinases in living cells, Nat Biotechnol 28, 743-747. [PubMed: 20581846]

[13]. Dagliyan O, Tarnawski M, Chu PH, Shirvanyants D, Schlichting I, Dokholyan NV, and Hahn KM (2016) Engineering extrinsic disorder to control protein activity in living cells, Science 354, 1441-1444. [PubMed: 27980211]

[14]. Dagliyan O, Shirvanyants D, Karginov AV, Ding F, Fee L, Chandrasekaran SN, Freisinger CM, Smolen GA, Huttenlocher A, Hahn KM, and Dokholyan NV (2013) Rational design of a ligandcontrolled protein conformational switch, Proc Natl Acad Sci U S A 110, 6800-6804. [PubMed: 23569285] 
[15]. Mobitz H, Jahnke W, and Cowan-Jacob SW (2017) Expanding the Opportunities for Modulating Kinase Targets with Allosteric Approaches, Curr Top Med Chem 17, 59-70. [PubMed: 27448652]

[16]. Monod J, Wyman J, and Changeux JP (1965) On the Nature of Allosteric Transitions: A Plausible Model, Journal of molecular biology 12, 88-118. [PubMed: 14343300]

[17]. Koshland DE Jr., Nemethy G, and Filmer D (1966) Comparison of experimental binding data and theoretical models in proteins containing subunits, Biochemistry 5, 365-385. [PubMed: 5938952]

[18]. Ackers GK (1998) Deciphering the molecular code of hemoglobin allostery, Adv Protein Chem 51, 185-253. [PubMed: 9615171]

[19]. Lipscomb WN (1994) Aspartate transcarbamylase from Escherichia coli: activity and regulation, Adv Enzymol Relat Areas Mol Biol 68, 67-151. [PubMed: 8154326]

[20]. Kemp RG, and Foe LG (1983) Allosteric regulatory properties of muscle phosphofructokinase, Mol Cell Biochem 57, 147-154. [PubMed: 6228716]

[21]. Goodsell DS, and Olson AJ (2000) Structural symmetry and protein function, Annu Rev Biophys Biomol Struct 29, 105-153. [PubMed: 10940245]

[22]. Stevens SY, Sanker S, Kent C, and Zuiderweg ER (2001) Delineation of the allosteric mechanism of a cytidylyltransferase exhibiting negative cooperativity, Nature structural biology 8, 947-952. [PubMed: 11685240]

[23]. Tzeng SR, and Kalodimos CG (2012) Protein activity regulation by conformational entropy, Nature 488, 236-240. [PubMed: 22801505]

[24]. Popovych N, Sun S, Ebright RH, and Kalodimos CG (2006) Dynamically driven protein allostery, Nature structural \& molecular biology 13, 831-838.

[25]. Freiburger L, Miletti T, Zhu S, Baettig O, Berghuis A, Auclair K, and Mittermaier A (2014) Substrate-dependent switching of the allosteric binding mechanism of a dimeric enzyme, Nat Chem Biol 10, 937-942. [PubMed: 25218742]

[26]. Capdevila DA, Braymer JJ, Edmonds KA, Wu H, and Giedroc DP (2017) Entropy redistribution controls allostery in a metalloregulatory protein, Proc Natl Acad Sci U S A 114, 4424-4429. [PubMed: 28348247]

[27]. Wang S, Thomas K, and Schramm VL (2014) Catalytic site cooperativity in dimeric methylthioadenosine nucleosidase, Biochemistry 53, 1527-1535. [PubMed: 24502544]

[28]. Koehn EM, Fleischmann T, Conrad JA, Palfey BA, Lesley SA, Mathews II, and Kohen A (2009) An unusual mechanism of thymidylate biosynthesis in organisms containing the thy $\mathrm{X}$ gene, Nature 458, 919-923. [PubMed: 19370033]

[29]. Mishanina TV, Yu L, Karunaratne K, Mondal D, Corcoran JM, Choi MA, and Kohen A (2016) An unprecedented mechanism of nucleotide methylation in organisms containing thyX, Science 351, 507-510. [PubMed: 26823429]

[30]. Anderson KS (2017) Understanding the molecular mechanism of substrate channeling and domain communication in protozoal bifunctional TS-DHFR, Protein Eng Des Sel 30, 253-261. [PubMed: 28338744]

[31]. Taddia L, D’ Arca D, Ferrari S, Marraccini C, Severi L, Ponterini G, Assaraf YG, Marverti G, and Costi MP (2015) Inside the biochemical pathways of thymidylate synthase perturbed by anticancer drugs: Novel strategies to overcome cancer chemoresistance, Drug resistance updates : reviews and commentaries in antimicrobial and anticancer chemotherapy 23, 20-54. [PubMed: 26690339]

[32]. Spencer HT, Villafranca JE, and Appleman JR (1997) Kinetic scheme for thymidylate synthase from Escherichia coli: determination from measurements of ligand binding, primary and secondary isotope effects, and pre-steady-state catalysis, Biochemistry 36, 4212-4222. [PubMed: 9100016]

[33]. Maley F, Pedersen-Lane J, and Changchien L (1995) Complete restoration of activity to inactive mutants of Escherichia coli thymidylate synthase: evidence that E. coli thymidylate synthase is a half-the-sites activity enzyme, Biochemistry 34, 1469-1474. [PubMed: 7849005]

[34]. Johnson EF, Hinz W, Atreya CE, Maley F, and Anderson KS (2002) Mechanistic characterization of Toxoplasma gondii thymidylate synthase (TS-DHFR)-dihydrofolate reductase. Evidence for a 
TS intermediate and TS half-sites reactivity, The Journal of biological chemistry 277, 4312643136. [PubMed: 12192007]

[35]. Dev IK, Dallas WS, Ferone R, Hanlon M, McKee DD, and Yates BB (1994) Mode of binding of folate analogs to thymidylate synthase. Evidence for two asymmetric but interactive substrate binding sites, The Journal of biological chemistry 269, 1873-1882. [PubMed: 8294436]

[36]. Lovelace LL, Gibson LM, and Lebioda L (2007) Cooperative inhibition of human thymidylate synthase by mixtures of active site binding and allosteric inhibitors, Biochemistry 46, 28232830. [PubMed: 17297914]

[37]. Reilly RT, Barbour KW, Dunlap RB, and Berger FG (1995) Biphasic binding of 5-fluoro-2' deoxyuridylate to human thymidylate synthase, Molecular pharmacology 48, 72-79. [PubMed: 7623777]

[38]. Arvizu-Flores AA, Sugich-Miranda R, Arreola R, Garcia-Orozco KD, Velazquez-Contreras EF, Montfort WR, Maley F, and Sotelo-Mundo RR (2008) Role of an invariant lysine residue in folate binding on Escherichia coli thymidylate synthase: calorimetric and crystallographic analysis of the K48Q mutant, The international journal of biochemistry \& cell biology 40, 22062217. [PubMed: 18403248]

[39]. Sapienza PJ, and Lee AL (2016) Widespread Perturbation of Function, Structure, and Dynamics by a Conservative Single-Atom Substitution in Thymidylate Synthase, Biochemistry 55, 57025713. [PubMed: 27649373]

[40]. Sapienza PJ, Falk BT, and Lee AL (2015) Bacterial Thymidylate Synthase Binds Two Molecules of Substrate and Cofactor without Cooperativity, J Am Chem Soc 137, 14260-14263. [PubMed: 26517288]

[41]. Stroud RM, and Finer-Moore JS (2003) Conformational dynamics along an enzymatic reaction pathway: thymidylate synthase, "the movie", Biochemistry 42, 239-247. [PubMed: 12525150]

[42]. Falk BT, Sapienza PJ, and Lee AL (2016) Chemical shift imprint of intersubunit communication in a symmetric homodimer, Proc Natl Acad Sci U S A.

[43]. Anderson AC, O’Neil RH, DeLano WL, and Stroud RM (1999) The structural mechanism for half-the-sites reactivity in an enzyme, thymidylate synthase, involves a relay of changes between subunits, Biochemistry 38, 13829-13836. [PubMed: 10529228]

[44]. Finer-Moore JS, Lee TT, and Stroud RM (2018) A Single Mutation Traps a Half-Sites Reactive Enzyme in Midstream, Explaining Asymmetry in Hydride Transfer, Biochemistry 57, 27862795. [PubMed: 29717875]

[45]. Wang Z, Sapienza PJ, Abeysinghe T, Luzum C, Lee AL, Finer-Moore JS, Stroud RM, and Kohen A (2013) $\mathrm{Mg} 2+$ binds to the surface of thymidylate synthase and affects hydride transfer at the interior active site, J Am Chem Soc 135, 7583-7592. [PubMed: 23611499]

[46]. Chen D, Jansson A, Sim D, Larsson A, and Nordlund P (2017) Structural analyses of human thymidylate synthase reveal a site that may control conformational switching between active and inactive states, The Journal of biological chemistry 292, 13449-13458. [PubMed: 28634233]

[47]. Hyatt DC, Maley F, and Montfort WR (1997) Use of strain in a stereospecific catalytic mechanism: crystal structures of Escherichia coli thymidylate synthase bound to FdUMP and methylenetetrahydrofolate, Biochemistry 36, 4585-4594. [PubMed: 9109668]

[48]. Sapienza PJ, and Lee AL (2014) Backbone and ILV methyl resonance assignments of E. coli thymidylate synthase bound to cofactor and a nucleotide analogue, Biomolecular NMR assignments 8, 195-199. [PubMed: 23653343]

[49]. Strop P, Changchien L, Maley F, and Montfort WR (1997) Crystal structures of a marginally active thymidylate synthase mutant, Arg 126-->Glu, Protein Sci 6, 2504-2511. [PubMed: 9416600]

[50]. Selvaratnam R, VanSchouwen B, Fogolari F, Mazhab-Jafari MT, Das R, and Melacini G (2012) The Projection Analysis of NMR Chemical Shifts Reveals Extended EPAC Autoinhibition Determinants, Biophysical Journal 102, 630-639. [PubMed: 22325287]

[51]. Boulton S, Akimoto M, Selvaratnam R, Bashiri A, and Melacini G (2014) A Tool Set to Map Allosteric Networks through the NMR Chemical Shift Covariance Analysis, Sci Rep-Uk 4. 
[52]. Sun H, Kay LE, and Tugarinov V (2011) An optimized relaxation-based coherence transfer NMR experiment for the measurement of side-chain order in methyl-protonated, highly deuterated proteins, The journal of physical chemistry. B 115, 14878-14884. [PubMed: 22040035]

[53]. Lakomek NA, Ying J, and Bax A (2012) Measurement of (1)(5)N relaxation rates in perdeuterated proteins by TROSY-based methods, Journal of biomolecular NMR 53, 209-221. [PubMed: 22689066]

[54]. Pronk S, Pall S, Schulz R, Larsson P, Bjelkmar P, Apostolov R, Shirts MR, Smith JC, Kasson PM, van der Spoel D, Hess B, and Lindahl E (2013) GROMACS 4.5: a high-throughput and highly parallel open source molecular simulation toolkit, Bioinformatics 29, 845-854. [PubMed: 23407358]

[55]. Hess B, Kutzner C, van der Spoel D, and Lindahl E (2008) GROMACS 4: Algorithms for Highly Efficient, Load-Balanced, and Scalable Molecular Simulation, J Chem Theory Comput 4, 435447. [PubMed: 26620784]

[56]. Best RB, Zhu X, Shim J, Lopes PE, Mittal J, Feig M, and Mackerell AD Jr. (2012) Optimization of the additive CHARMM all-atom protein force field targeting improved sampling of the backbone phi, psi and side-chain chi(1) and chi(2) dihedral angles, J Chem Theory Comput 8 , 3257-3273. [PubMed: 23341755]

[57]. Yu W, He X, Vanommeslaeghe K, and MacKerell AD Jr. (2012) Extension of the CHARMM General Force Field to sulfonyl-containing compounds and its utility in biomolecular simulations, J Comput Chem 33, 2451-2468. [PubMed: 22821581]

[58]. Vanommeslaeghe K, Hatcher E, Acharya C, Kundu S, Zhong S, Shim J, Darian E, Guvench O, Lopes P, Vorobyov I, and Mackerell AD Jr. (2010) CHARMM general force field: A force field for drug-like molecules compatible with the CHARMM all-atom additive biological force fields, J Comput Chem 31, 671-690. [PubMed: 19575467]

[59]. Proctor EA, Kota P, Aleksandrov AA, He L, Riordan JR, and Dokholyan NV (2015) Rational Coupled Dynamics Network Manipulation Rescues Disease-Relevant Mutant Cystic Fibrosis Transmembrane Conductance Regulator, Chem Sci 6, 1237-1246. [PubMed: 25685315]

[60]. Stout TJ, Sage CR, and Stroud RM (1998) The additivity of substrate fragments in enzymeligand binding, Structure 6, 839-848. [PubMed: 9687366]

[61]. Fenton AW (2008) Allostery: an illustrated definition for the 'second secret of life', Trends Biochem Sci 33, 420-425. [PubMed: 18706817]

[62]. Cui DS, Beaumont V, Ginther PS, Lipchock JM, and Loria JP (2017) Leveraging Reciprocity to Identify and Characterize Unknown Allosteric Sites in Protein Tyrosine Phosphatases, Journal of molecular biology 429, 2360-2372. [PubMed: 28625849]

[63]. Lee AL, and Sapienza PJ (2018) Thermodynamic and NMR Assessment of Ligand Cooperativity and Intersubunit Communication in Symmetric Dimers: Application to Thymidylate Synthase, Front Mol Biosci 5, 47. [PubMed: 29888227]

[64]. Byun JA, and Melacini G (2018) NMR methods to dissect the molecular mechanisms of diseaserelated mutations (DRMs): Understanding how DRMs remodel functional free energy landscapes, Methods.

[65]. Carreras CW, and Santi DV (1995) The catalytic mechanism and structure of thymidylate synthase, Annu Rev Biochem 64, 721-762. [PubMed: 7574499]

[66]. Kanaan N, Marti S, Moliner V, and Kohen A (2009) QM/MM study of thymidylate synthase: enzymatic motions and the temperature dependence of the rate limiting step, The journal of physical chemistry. A 113, 2176-2182. [PubMed: 19182971]

[67]. Hunenberger PH, Mark AE, and van Gunsteren WF (1995) Fluctuation and cross-correlation analysis of protein motions observed in nanosecond molecular dynamics simulations, Journal of molecular biology 252, 492-503. [PubMed: 7563068]

[68]. Matthews DA, Appelt K, and Oatley SJ (1989) Stacked beta-bulges in thymidylate synthase account for a novel right-handed rotation between opposing beta-sheets, Journal of molecular biology 205, 449-454. [PubMed: 2648002]

[69]. Fenwick RB, Orellana L, Esteban-Martin S, Orozco M, and Salvatella X (2014) Correlated motions are a fundamental property of beta-sheets, Nat Commun 5, 4070. [PubMed: 24915882] 
[70]. Clore GM, and Schwieters CD (2004) Amplitudes of protein backbone dynamics and correlated motions in a small alpha/beta protein: correspondence of dipolar coupling and heteronuclear relaxation measurements, Biochemistry 43, 10678-10691. [PubMed: 15311929]

[71]. Fadel AR, Jin DQ, Montelione GT, and Levy RM (1995) Crankshaft motions of the polypeptide backbone in molecular dynamics simulations of human type-alpha transforming growth factor, Journal of biomolecular NMR 6, 221-226. [PubMed: 8589611]

[72]. Fenwick RB, Esteban-Martin S, Richter B, Lee D, Walter KF, Milovanovic D, Becker S, Lakomek NA, Griesinger C, and Salvatella X (2011) Weak long-range correlated motions in a surface patch of ubiquitin involved in molecular recognition, J Am Chem Soc 133, 10336-10339. [PubMed: 21634390]

[73]. Emberly EG, Mukhopadhyay R, Tang C, and Wingreen NS (2004) Flexibility of beta-sheets: principal component analysis of database protein structures, Proteins 55, 91-98. [PubMed: 14997543]

[74]. Variath P, Liu Y, Lee TT, Stroud RM, and Santi DV (2000) Effects of subunit occupancy on partitioning of an intermediate in thymidylate synthase mutants, Biochemistry 39, 2429-2435. [PubMed: 10704192] 
A

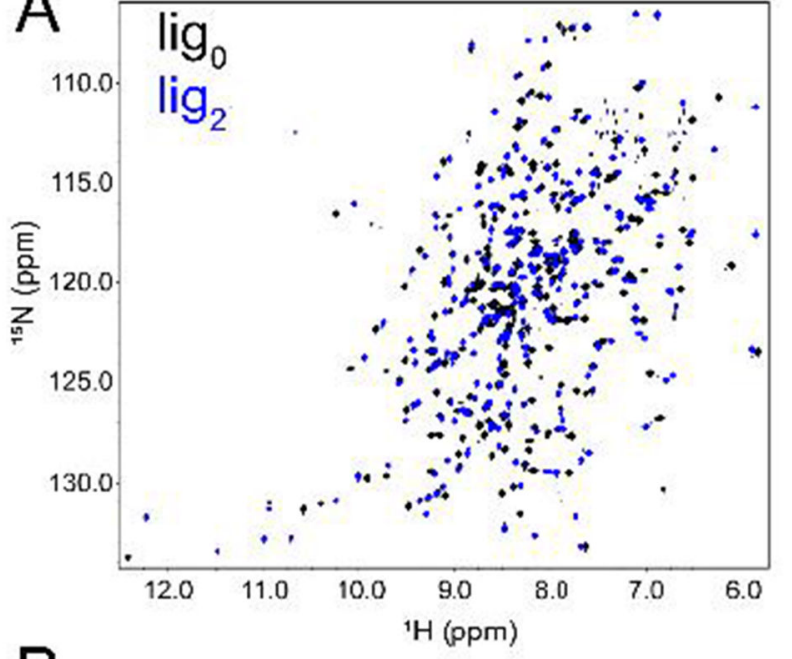

C

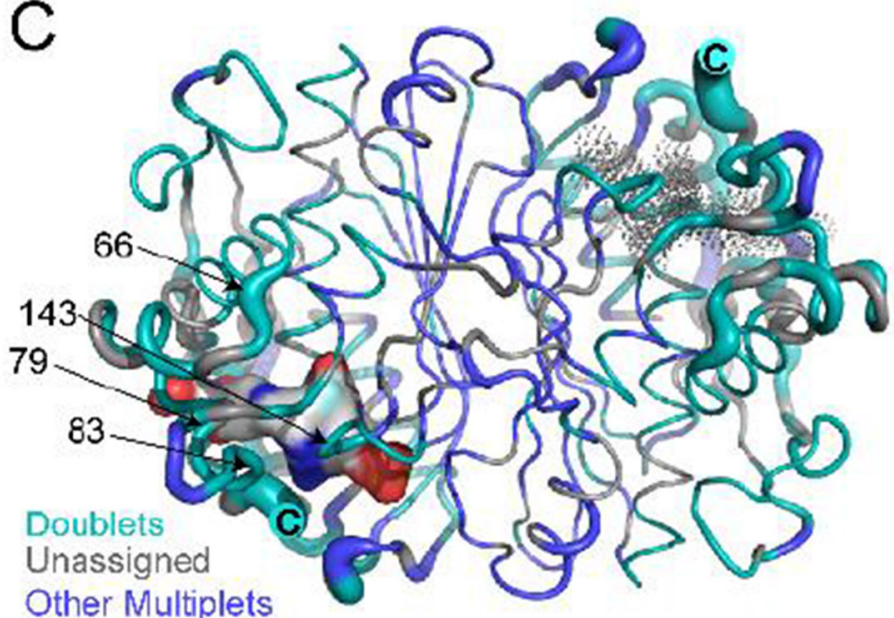

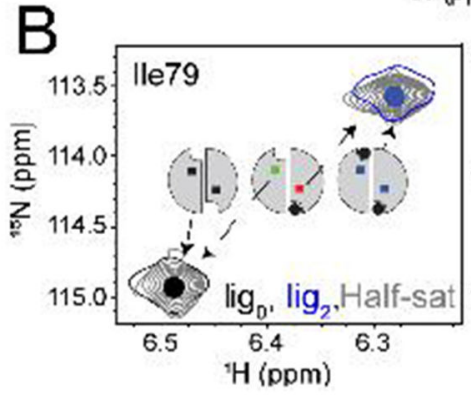
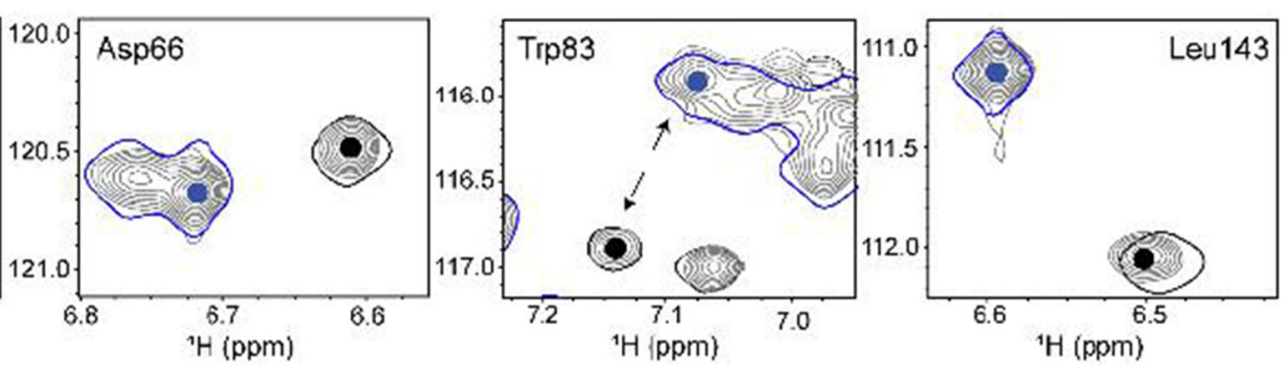

Figure 1.

Some distal sites are insulated from effects of diligand binding to local subunit. A) Overlay of Apo (lig, , black) and diligand (lig 2 , blue) TROSY ${ }^{1} \mathrm{H}^{-15} \mathrm{~N}$ HSQCs show single sets of peaks for both states. B) Spectra at midpoint of diligand titration (gray) are compared with spectra of free and diligand saturated titration endpoints in black and blue, respectively.

These are examples where resonances from free and bound subunits of the $\operatorname{lig}_{1}$ state overlap with those of the $\operatorname{lig}_{0}$ and $\operatorname{lig}_{2}$ states respectively, indicating that for these amides, the distal subunit is unaffected by local binding events. Dots identify peaks originating from the panel title amino acid as a way to discriminate from contours derived from other residues. C) Putty width of the $\operatorname{lig}_{2} \mathrm{X}$-ray model is scaled to the $\mathrm{C}^{\mathrm{a}}-\mathrm{C}^{\mathrm{a}}$ coordinate difference between aligned $\operatorname{lig}_{0}(2 \mathrm{FTQ})$ and $\operatorname{lig}_{2}(1 \mathrm{TSN})$ crystal structures. Amides giving doublets at intermediate titration points are in aqua, other multiplets are blue, and prolines and unassigned residues are gray. Note the $\mathrm{C}$-terminus, which undergoes the largest conformational change, is dominated by the doublet pattern indicating this conformational change is strictly coupled to binding in the local subunit. The bound ligand is shown in vdw surface and the empty subunit is denoted by stippled spheres. 

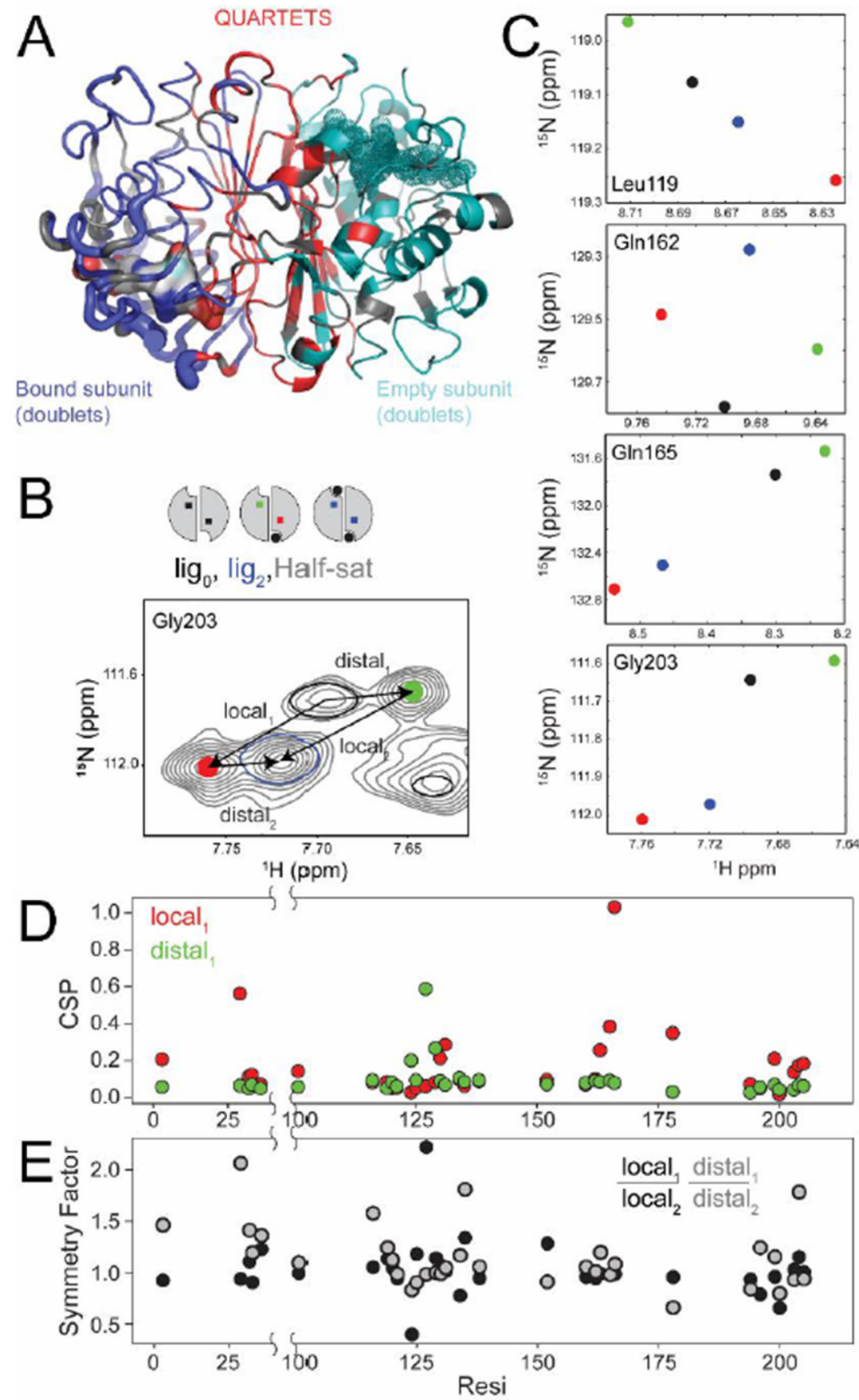

Figure 2.

Diligand binding events are communicated to distal subunits. A) $\operatorname{Lig}_{2} \mathrm{X}$-ray model putty width is scaled to the $\mathrm{C}^{\mathrm{a}}-\mathrm{C}^{\mathrm{a}}$ coordinate difference between aligned $\operatorname{lig}_{0}$ and $\operatorname{lig}_{2}$ structures (left, bound subunit only). The dimer is depicted to show the $\operatorname{lig}_{1}$ state with the filled subunit on the left having doublets colored blue and the empty subunit on the right with doublets in aqua. Residues with quartet patterns at intermediate diligand titration points are colored in red in both subunits. Note these do not track with conformational change linked to binding, rather they cluster to the $\beta$-sheet making up the dimer interface and connecting the two 
active sites. B) Example of a quartet pattern in a half saturated diligand TROSY ${ }^{1} \mathrm{H}_{-}{ }^{15} \mathrm{~N}$ HHSQC. The manifold of states are shown in the schematic. For reference, $\operatorname{lig}_{0}$ and $\operatorname{lig}_{2}$ HSQCs are shown with single contour lines in black and blue respectively. Resonances from the free and bound subunit of the $\operatorname{lig}_{1}$ state are identified with green and red dots. Note the vectors used to define the chemical shift perturbations associated with the two binding events as sensed by the local and distal subunits. C) HSQC coordinates for typical quartet resonances. Dots rather than contour plots are shown for simplicity and are colored as in panel B. D) CSPs associated with the first diligand binding event as sensed by the local (red) and distal (green) sites. E) Measurement of allosteric reciprocity based on ratios of two local and two distal CSPs (see panel B) in black and gray respectively. Ratios of one indicate perfect reciprocity. 


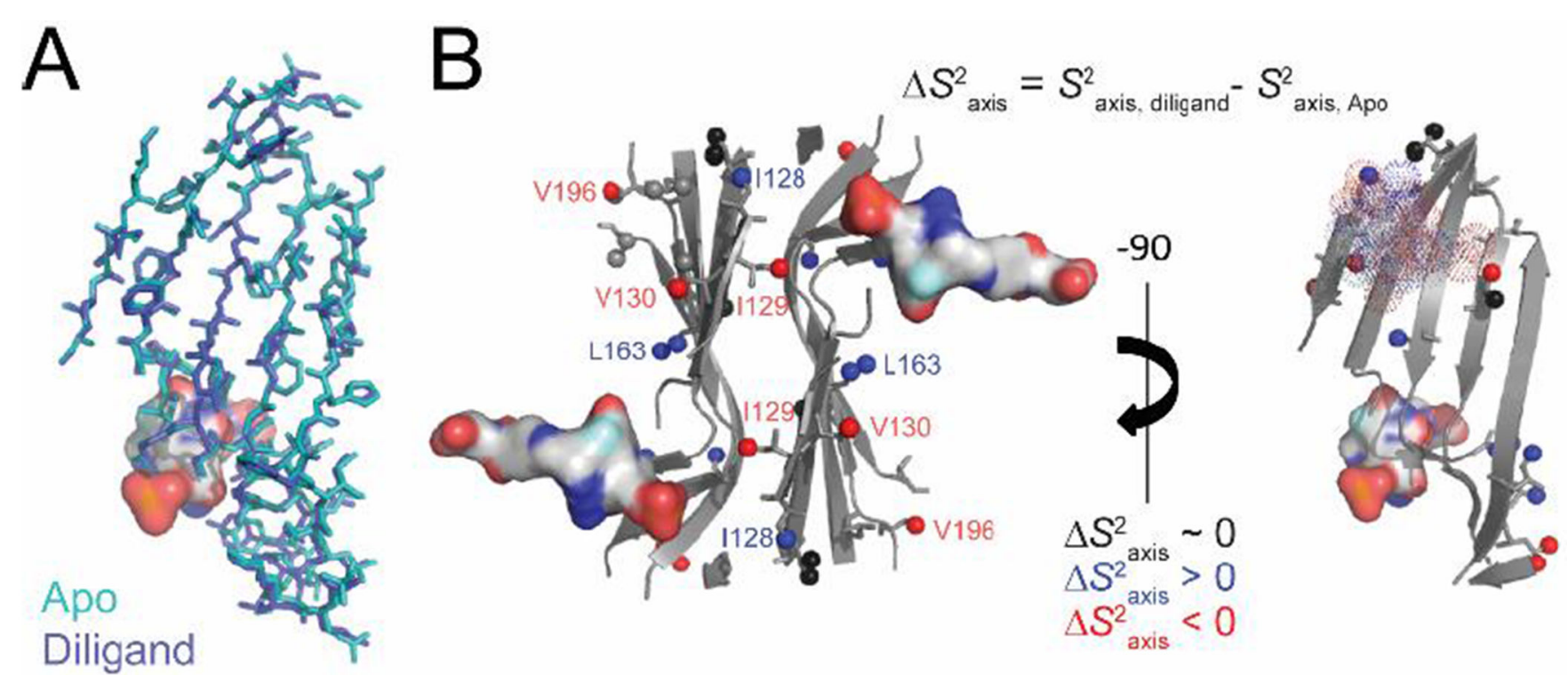

Figure 3.

Structural and dynamic comparison of $\operatorname{lig}_{0}$ and $\operatorname{lig}_{2}$ dimer interface $\beta$-sheets. A) Alignment (one subunit only) of the $\operatorname{lig}_{0}$ (aqua) and $\operatorname{lig}_{2}$ (blue) sheets shows they are nearly identical with an all atom RMSD of $0.27 \AA$. Single diligand molecule is shown in surface. B) NMR order parameter, $S^{2}{ }_{\text {axis }}$, shows differences in methyl group ps-ns dynamics among the $\operatorname{lig}_{0}$ and $\operatorname{lig}_{2}$ states. Differences in $\beta$-sheet methyl groups are highlighted. Probes that become more rigid upon diligand binding are in blue, probes that become more flexible are in red, and unchanged methyl groups are colored black. Probes without measurements in one or both states are colored gray. In the left panel, both diligand molecules are shown in surface. In the right, rotated panel, the sheet from one subunit is stripped away and the diligand is shown in dots for clarity. 




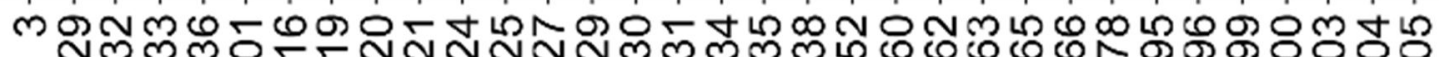

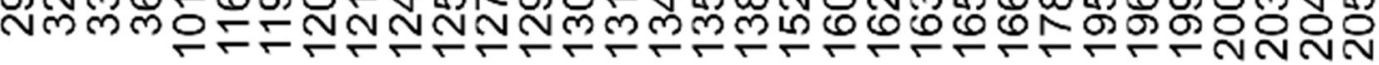
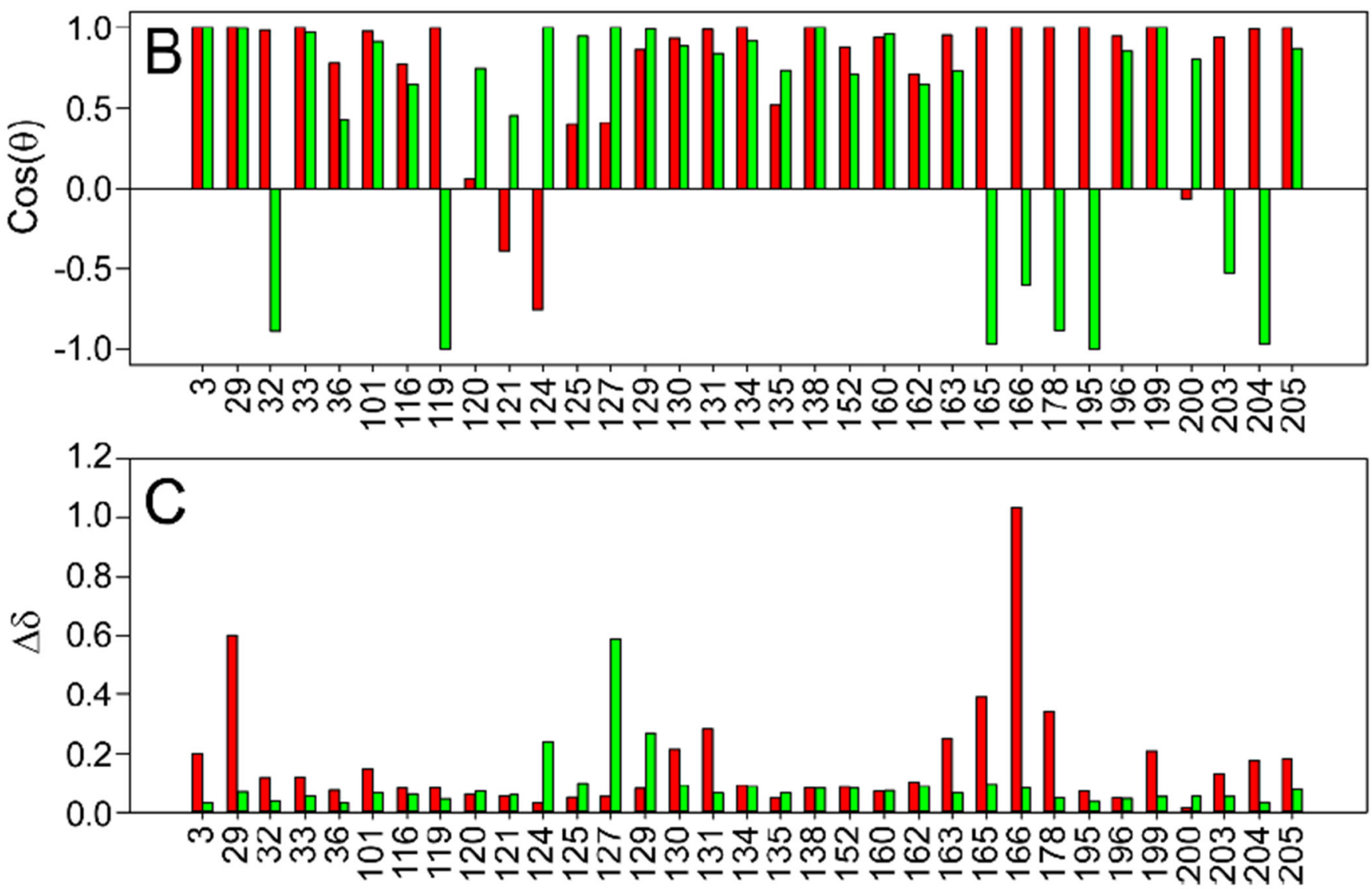

Figure 4.

Chemical shift projection analysis (CHESPA) shows complex behavior of diligand quartet residues. Fractional shift ( $\chi$, Panel A) and $\cos (\theta$, Panel B) values show the magnitude and direction of singly bound, $\operatorname{lig}_{1}$ resonances relative to the vector connecting $\operatorname{lig}_{0}$ and $\operatorname{lig}_{2}$ peaks (see Scheme 1 in methods). Data for the bound and free $\operatorname{lig}_{1}$ resonances are shown in red and green respectively. Note the variability in the magnitude and direction of both CHESPA parameters, which argues against $\operatorname{lig}_{1}$ binding causing a global concerted shift in the populations of a dynamic two state equilibrium. Panel C, simply shows the overall 
chemical shift perturbation (see methods) of the two $\operatorname{lig}_{1}$ peaks for appreciation of the magnitude of the shifts. 

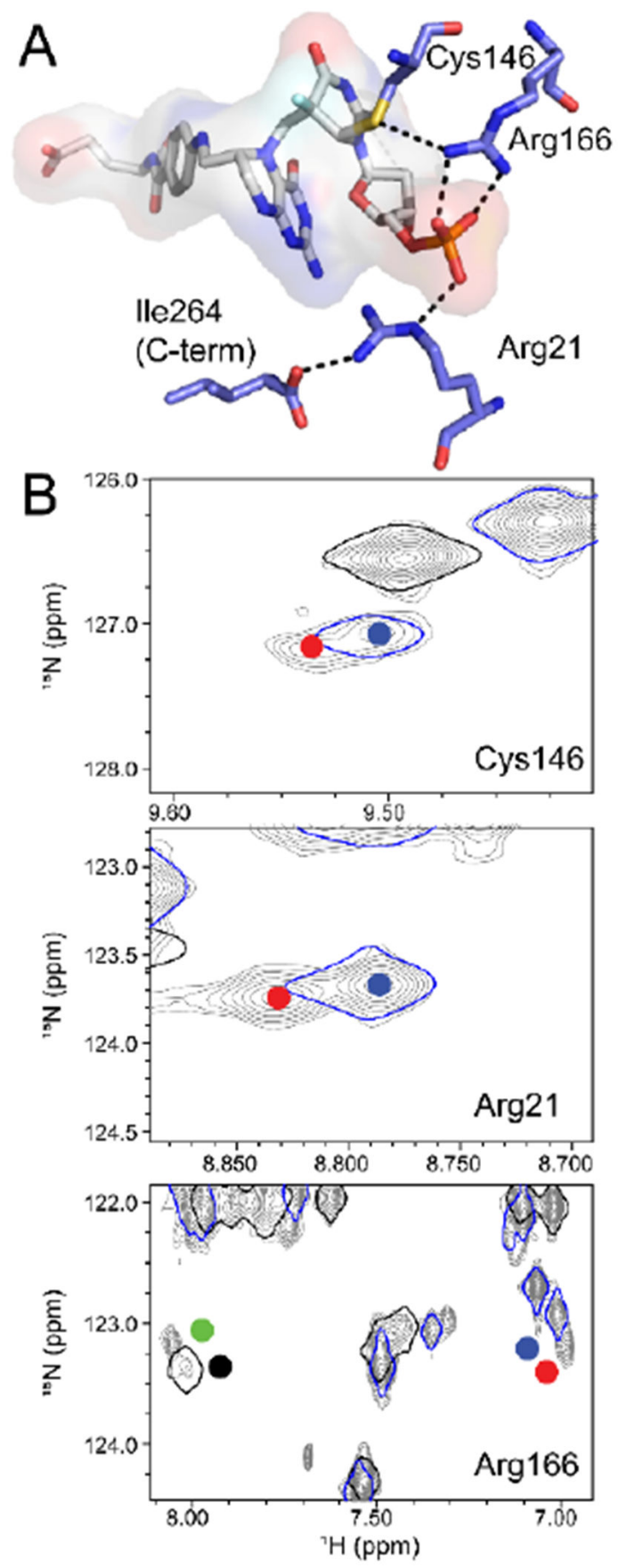

Figure 5.

Effects of diligand binding are propagated to key active site residues in the distal subunit. A) Close up of active site residues Arg21, Cys146, and Arg 166 whose chemical shift patterns indicate inte-ractive site communication. The $\mathrm{C}$-terminal residue 264 is shown to emphasize the bi-functional role of Arg21 in contacting dUMP and stabilizing the closed conformation of the C-terminus. B) TROSY ${ }^{1} \mathrm{H}^{-15} \mathrm{~N}$ HSQCs of $\operatorname{lig}_{0}$ (black, single contour line), lig 2 (blue, single contour line), and half saturated with diligand (gray). Dots represent the different microstates with $\operatorname{lig}_{0}$ in black, $\operatorname{lig}_{2}$ in blue, $\operatorname{lig}_{1}$ free subunit in green, and $\operatorname{lig}_{1}$ bound subunit 
in red. Note resonances for $\operatorname{Arg} 21$ and Cys146 are broadened away in the $\operatorname{lig}_{0}$ spectrum, and are therefore likely not visible for the $\operatorname{lig}_{1}$ free state. 
A
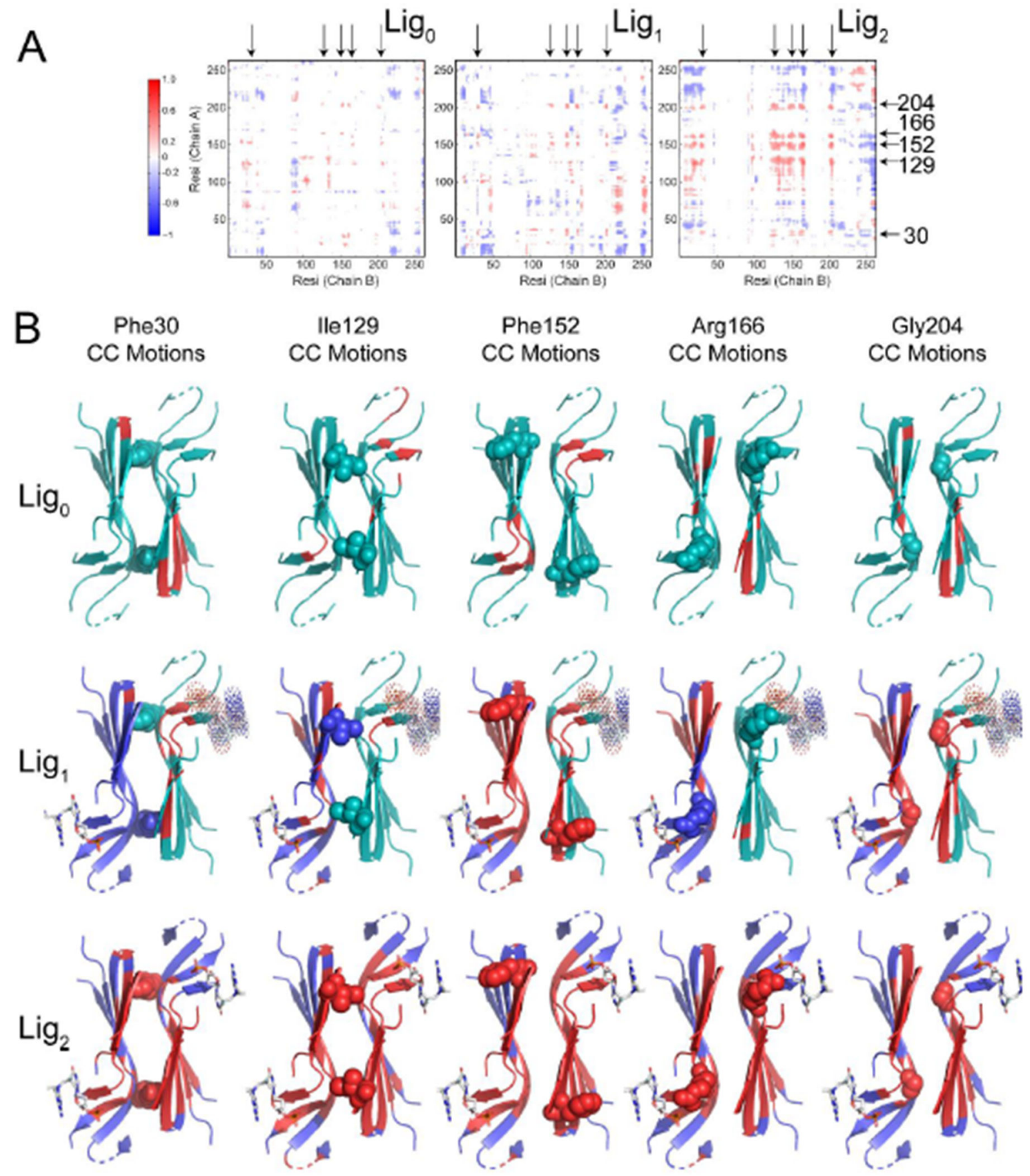

Figure 6.

Inter-subunit dynamic coupling mediated by the dimer interface $\beta$-sheet. The three panels in vertical panel A show inter-subunit $\mathrm{C}^{\mathrm{a}}$ cross-correlated motion matrices calculated from MD simulations of $\operatorname{lig}_{0}$ (left), $\operatorname{lig}_{1}$ (middle), and $\operatorname{lig}_{2}$ (right) states. A z-score cutoff of 1.5 was used such that correlations below this signal-to-noise threshold were set to zero.

Correlated motions are red and anti-correlated motions are blue. For the $\operatorname{lig}_{1}$ case, the bound subunit is chain A (y-axis). Five hotspot residues identified in the $\operatorname{lig}_{2}$ simulations are marked by arrows. In panel B, inter-subunit cross-correlated motions involving hotspot 
residues Phe30, Ile129, Phe152, Arg166, and Gly204 are shown. Data from $\operatorname{lig}_{0}, \operatorname{lig}_{1}$, and $\operatorname{lig}_{2}$ simulations are in rows one, two, and three respectively. In the $\operatorname{lig}_{1}$ row, the diligand in the bound and un-bound subunits are shown in sticks and dots, respectively. Both diligands are displayed as sticks in the $\operatorname{lig}_{2}$ row. These correlations are extracted from the row and column vectors of the matrices marked by arrows in panel A. The dimer interface $\beta$-sheet connecting the two active sites are shown, with the hotspot residue in spacefill, empty subunits in teal, and bound subunits in blue. Residues with motions correlated to the particular hot spot residue are shown in red. For the asymmetrical singly bound state, the residues in the empty subunit that are correlated with a given hotspot residue in the bound subunit are colored red in the right protomer of the dimer (based on row vectors from the matrix) and residues in the bound subunit that are correlated with a given hotspot residue in the empty subunit are red in left protomer (based on row vectors from the matrix). 




Figure 7.

Cross-correlated dynamic hotspots map to the $\beta$-bulge within dimer interface sheet. A)

Orthogonal views of the dimer interface $\beta$-sheet are shown with one subunit in blue and the other in teal. Only the FdUMP moiety of diligand is shown for clarity. Note three dynamic hotspot residues, Phe30, Arg166, and Gly204 in gray lie at the vertex of the stacked $\beta$-bulge which stabilizes the $\sim 90^{\circ}$ right handed bend in the sheet. B) Main chain hydrogen bonds that stabilize the bulge are shown along with contacts between the bulge and diligand FdUMP which likely activate the network of inter-active site cross-correlated motions. C) $\beta$-bulge residues are physically coupled to other hotspots (Ile129 and Phe152) through inter-strand packing interactions, providing a mechanism for communication across the subunits at both ends of the sheet. 


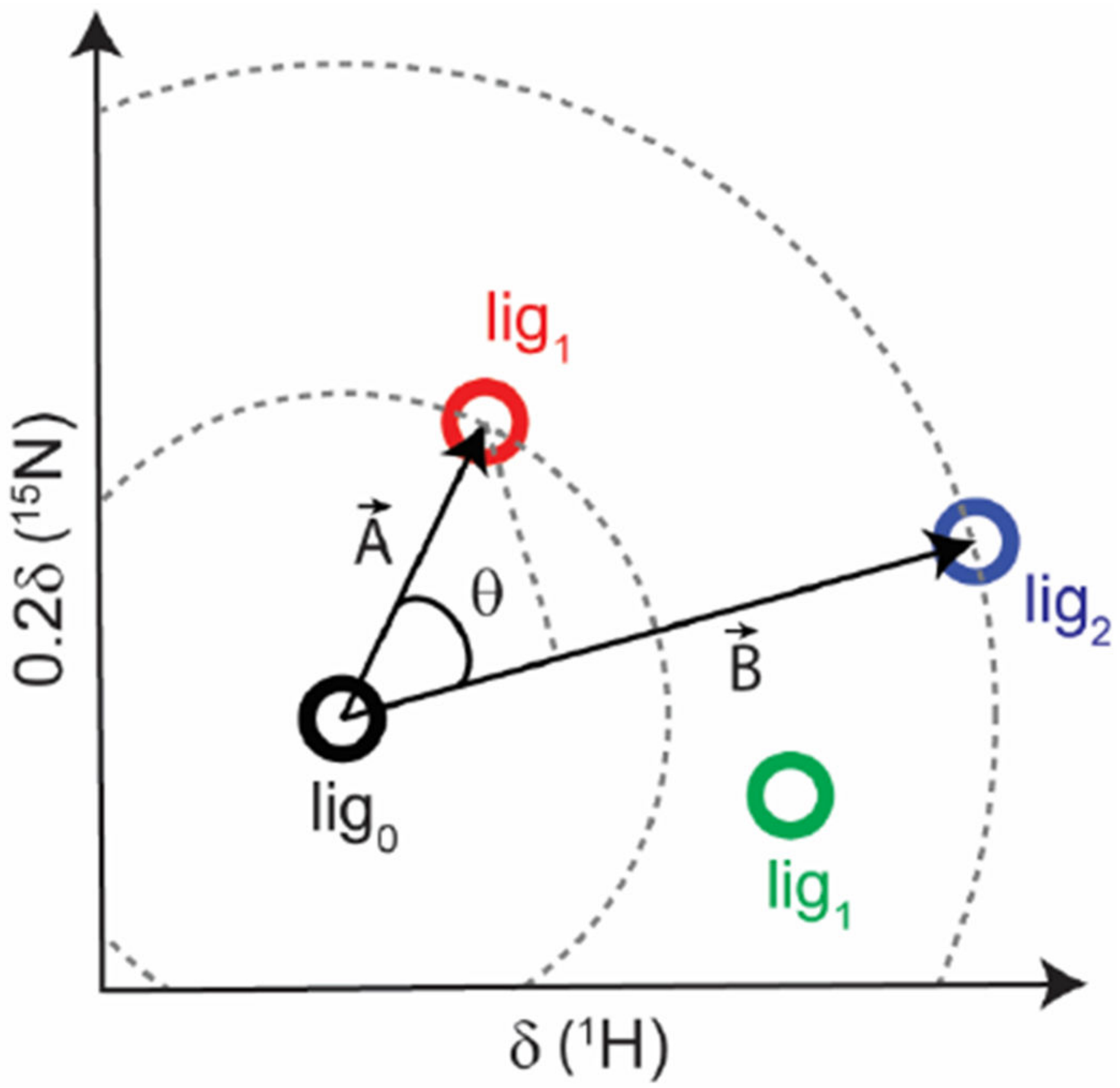

Scheme 1.

CHESPA Analysis of Quartet Resonance Patterns 